Pacific Journal of Mathematics

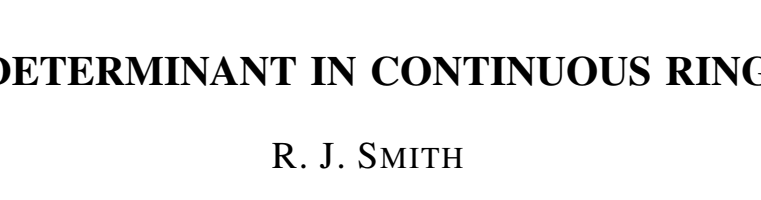




\title{
A DETERMINANT IN CONTINUOUS RINGS
}

\author{
R. J. SMITH
}

1. Introduction. In the theory, developed by Dieudonné [1], of determinants of nonsingular square matrices over a noncommutative field $K$ the determinantal values are cosets modulo the commutator subgroup of $K^{\times}$, the multiplicative group of $K$. Since the matrix groups $M_{n}^{\times}(K)$ and their commutator subgroups $C_{n}$ have the property that $M_{n}^{\times}(K) / C_{n}$ is independent of $n$, the latter cosets will serve just as well for determinantal values, at least for theorems involving only the multiplication of determinants.

The rings whose principal right ideal lattices form continuous geometries have many resemblances to matrix rings; in fact, the axioms of Continuous Geometry are satisfied by finite dimensional geometries over a field which are always equivalent to the right ideal lattice of some matrix ring. Irrespective of questions as to the existence or otherwise of fields in connection with a general continuous geometry playing a similar role to that of the field of coordinate values in the finite dimensional case we will show that multiplicative determinantal theorems can be obtained for the more general ring; the determinants will be cosets of the group of invertible ring elements modulo the closure of its commutator subgroup with respect to the rank-distance topology in the ring.

The definition of a complete rank ring is given by von Neumann [3, (iv)]. Essential properties of such a ring $\Re$ and the associated lattice of principal right ideals have been developed by von Neumann [3, 4] and Ehrlich [2]. We will assume throughout that $\mathfrak{R}$ is a complete rank ring, of characteristic not 2; and that if the discrete case (matrices over a field) applies, then the order of the matrices is at least 3.

2. Groups in a complete rank ring. Using a notation similar to that of [2], [3] we denote by $\&$ the group of invertible ring elements; that is, $u \in \mathfrak{\complement} \subset \Re$ if and only if the rank $R(u)$ of $u$ is 1 .

Definition 1 . We denote by $\Omega$ the closure of the commutator subgroup of $\xi$ in the rank-distance topology and by $\Omega^{\dagger}$ the closure of the group generated by the elements of class 2 in $(5$.

COROLlary 1 . $\Omega$ and $\Omega^{\dagger}$ are groups.

Received June 19, 1957. 
Proof. Let $\left\{t_{n} ; t_{n} \in \mathfrak{\xi}, n=1,2, \cdots\right\}$ be a converging sequence in $\Re$. Then $\lim _{n, m \rightarrow \infty} R\left(t_{n}-t_{m}\right)=0$ implies

$$
\lim _{n, m \rightarrow \infty} R\left(t_{n}^{-1}-t_{m}^{-1}\right)=\lim _{n, m \rightarrow \infty} R\left\{t_{n}^{-1}\left(t_{m}-t_{n}\right) t_{m}^{-1}\right\}=0
$$

and hence $\lim _{n \rightarrow \infty} t_{n}^{-1}$ exists in $\Re$. By the continuity of multiplication $\left(\lim _{n \rightarrow \infty} t_{n}\right)\left(\lim _{n \rightarrow \infty} t_{n}^{-1}\right)=1$ so that $\lim _{n \rightarrow \infty} t_{n}^{-1} \in \mathbb{8}$. The result then follows routinely after the observation that the inverse of a commutator is a commutator and the inverse of the general class 2 element $1+r\left(r^{2}=0\right)$ is $1-r$, also of class 2 .

Lemma 1. Let $t \in C^{2}$ (be of class 2), $s \in \mathbb{[}$. Then $s t s^{-1} \in C^{2}$.

Corollary 2. Let $t \in C^{2}, s \in \mathbb{F}$. Then $s t=t_{1} s$ for some $t_{1} \in C^{2}$.

Definition 2. We writh $u \cong s$ for nonsingular (invertible) $u, s \in \Re$ when $u=t s$ for some $t \in \Omega^{\dagger}$.

COROLLARY 3. The relation $\cong$ is an equivalence relation.

Lemma 2. Let $e$ be any idempotent of rank $1 / 2$ and $s$ be nonsingular and otherwise arbitrary in $\Re$. Then for some $t \in \Re$

$$
s \cong e+(1-e) t(1-e) .
$$

Proof. The existence of idempotents of rank 1/2 is assumed in continuous rings, that is, when the range of $R$ is the unit interval. In the discrete case the result has no meaning if the order of the matrices is odd.

Now suppose the principal left ideal $((1-e) s e)_{l}=\left(g_{1}\right)_{l}$ where $g_{1}=e g_{1} e$, $g_{1}^{2}=g_{1}[4$, Chapter 15]. By the Pierce decomposition, $s$ is the sum of the quantities in the blocks of

$$
\left[\begin{array}{ccc}
g_{1} s g_{1} & g_{1} s\left(e-g_{1}\right) & e s(1-e) \\
\left(e-g_{1}\right) s g_{1} & \left(e-g_{1}\right) s\left(e-g_{1}\right) & \\
(1-e) s g_{1} & (1-e) s\left(e-g_{1}\right) & (1-e) s(1-e)
\end{array}\right]
$$

where a matrix notation is used for clarity and to permit the comparison of later processes with standard matrix ones; we will simply equate such a partitioned array to the sum of its members. We have

$$
g_{1}=y_{1}(1-e) s e=y_{1}(1-e) s e g_{1}=y_{1}(1-e) s g_{1}
$$


for some $y_{1} \in \Re$ so that

$$
\begin{aligned}
& \left\{1+g_{1}\left(g_{1}-g_{1} s g_{1}\right) y_{1}(1-e)\right\} s \\
& \quad=\left[\begin{array}{ccc}
g_{1} & g_{1} s\left(e-g_{1}\right) & g_{1} s^{*}(1-e) \\
\left(e-g_{1}\right) s g_{1} & \left(e-g_{1}\right) s\left(e-g_{1}\right) & \left(e-g_{1}\right) s(1-e) \\
(1-e) s g_{1} & 0 & (1-e) s(1-e)
\end{array}\right]
\end{aligned}
$$

for some $s^{*} \in \Re$ since

$$
g_{1} s g_{1}+\left(g_{1}-g_{1} s g_{1}\right) y_{1}(1-e) s g_{1}=g_{1} s g_{1}+g_{1}-g_{1} s g_{1}=g_{1}
$$

and

$$
(1-e) s\left(e-g_{1}\right)=(1-e) s e-(1-e) s g_{1}=(1-e) s e g_{1}-(1-e) s g_{1}=0 .
$$

Multiplying on the left by $\left(1-(1-e) s g_{1}\right)\left(1-\left(e-g_{1}\right) s g_{1}\right)$ and on the right by $\left(1-g_{1} s\left(e-g_{1}\right)\right)\left(1-g_{1} s^{*}(1-e)\right)$ gives

$$
t_{1} s=\left[\begin{array}{ccc}
g_{1} & 0 & 0 \\
0 & \left(e-g_{1}\right) s_{1}\left(e-g_{1}\right) & \left(e-g_{1}\right) s_{1}(1-e) \\
0 & (1-e) s_{1}\left(e-g_{1}\right) & (1-e) s_{1}(1-e)
\end{array}\right]=s_{1}
$$

for some $s_{1} \in \Re$ and some $t_{1} \in \Re^{\dagger}$ by Corollary 2 .

Define $g_{n+1}, s_{n+1}, t_{n+1}$ for $n=1,2, \cdots$ as follows.

Let $\left((1-e) s_{n}\left(e-g_{1}-\cdots-g_{n}\right)\right)_{l}=\left(g_{n+1}\right)_{l}$ where $g_{n+1}^{2}=g_{n+1}$ and $\left(e-g_{1}-\right.$ $\left.\cdots-g_{n}\right) g_{n+1}\left(e-g_{1}-\cdots-g_{n}\right)=g_{n+1}$. We have, similarly to the above, the existence of a $t_{n+1} \in \Re^{\dagger}$ and an $s_{n+1} \in \Re$ such that

$$
t_{n+1} s=\left[\begin{array}{cc}
g_{1} \cdot g_{n} & 0 \\
0 & \left(e-g_{1}-\cdots-g_{n+1}\right) s_{n+1}\left(e-g_{1}-\cdots-g_{n+1}\right) \\
& (1-e) s_{n+1}\left(e-g_{1}-\cdots-g_{n+1}\right) \\
0 \\
\left(e-g_{1}-\cdots-g_{n+1}\right) s_{n+1}(1-e) \\
(1-e) s_{n+1}(1-e)
\end{array}\right]=s_{n+1} .
$$

Now,

$$
\frac{1}{2} \geqq R\left(g_{1}+\cdots+g_{n}\right)=R\left(g_{1}\right)+\cdots+R\left(g_{n}\right)=\sum_{i=1}^{n} R\left((1-e) s_{i}\left(e-g_{1}-\cdots-g_{i}\right)\right)
$$

so $\lim _{i \rightarrow \infty} R\left((1-e) s_{i}\left(e-g_{1}-\cdots-g_{i}\right)\right)=0$ and in turn 


$$
\lim _{i \rightarrow \infty}(1-e) s_{i}\left(e-g_{1}-\cdots-g_{i}\right)=0
$$

More strongly,

$$
\lim _{n, p \rightarrow \infty} R\left(g_{n+1}+\cdots+g_{n+p}\right)=\lim _{n, p \rightarrow \infty}\left\{R\left(g_{n+1}\right)+\cdots+R\left(g_{n+p}\right)\right\}=0 .
$$

Hence, by [3, (iv), Section 3] $\lim _{n \rightarrow \infty}\left(g_{1}+\cdots+g_{n}\right)=g$, say, exists in $\Re$; also, by the continuity of multiplication, $g=e g e$ and $g$ is idempotent, being the limit of a sequence of idempotents.

In order to prove that $\lim _{n \rightarrow \infty} t_{n}$ exists in $\Re$ and so belongs to $\Re^{\dagger}$ we note that

$$
\begin{aligned}
& \left(1-(1-e) s_{n} g_{n+1}\right)\left(1-\left(e-g_{1}-\cdots-g_{n+1}\right) s_{n} g_{n+1}\right) \\
\cdot & \left(1+g_{n+1}\left(g_{n+1}-g_{n+1} s_{n} g_{n+1}\right) y_{n+1}(1-e)\right) t_{n} s \\
\cdot & \left(1-g_{n+1} s_{n}\left(e-g_{1}-\cdots-g_{n+1}\right)\right)\left(1-g_{n+1} s_{n}^{*}(1-e)\right)=t_{n+1} s
\end{aligned}
$$

where $s_{n}^{*} \in \Re$ and $y_{n+1}$ is defined by the condition $g_{n+1}=y_{n+1}(1-e) s_{n} e$ The last two factors on the left side of (2) may be transferred after a similarity transformation to the left of $t_{n} s$, by Corollary 2, giving

$$
\left(1+\Phi\left(g_{n+1}\right)\right) t_{n} s=t_{n+1} s
$$

where $\Phi\left(g_{n+1}\right)$ is an expression involving no more than $2^{5}-1=31$ terms, each containing $g_{n+1}$ as a factor and so of rank $\leqq R\left(g_{n+1}\right)$. Hence $t_{n+1}$ $-t_{n}=\Phi\left(g_{n+1}\right) t_{n}$ and

$$
\begin{aligned}
R\left(t_{n+1}-t_{n}\right) & \leqq R \Phi\left(g_{n+1}\right) \leqq 31 R\left(g_{n+1}\right), \\
R\left(t_{n+p}-t_{n}\right) & \leqq \sum_{i=1}^{p} R\left(t_{n+i}-t_{n+i-1}\right) \\
& \leqq 31 \sum_{i=1}^{p} R\left(g_{n+i}\right) \rightarrow 0 \text { as } n, p \rightarrow \infty .
\end{aligned}
$$

[3, (iv), Equation 3, (iii)]

We conclude that

$$
\lim _{n \rightarrow \infty}\left(1-g_{1}-\cdots-g_{n}\right) s_{n}\left(1-g_{1}-\cdots-g_{n}\right)=\lim _{n \rightarrow \infty}\left(t_{n} s-\left(g_{1}+\cdots+g_{n}\right)\right)
$$

exists in $\Re$. It equals $(1-g) t(1-g)$ for some $t \in \Re$. Moreover, $(1-e)$ $\cdot t(e-g)=0$ by $(1)$. Then

$$
s \cong\left[\begin{array}{ccc}
g & 0 & 0 \\
0 & (e-g) t(e-g) & (e-g) t(1-e) \\
0 & 0 & (1-e) t(1-e)
\end{array}\right]
$$


where $R((e-g) t(e-g)) \leqq 1 / 2$ and $(e-g) t(e-g)$ has an inverse in the subring $\Re(e-g)$.

By the proof of $[4$, Lemma 3.6], if $(1-e) h(1-e)=h$ is an idempotent of rank equal to $R(e-g)$, then $e-g, h$ define quantities $x, y \in \Re$ such that

$$
x h=(e-g) x=x, \quad h y=y(e-g)=y, \quad x y=e-g, \quad y x=h .
$$

We have that $1+x, 1+y \in C^{2}$ since $x^{2}=x h(e-g) x=0, y^{2}=y(e-g) h y=0$, and so $(1+x)(1-y)(1+x)=1-(e-g)-h+x-y \in \Omega^{\dagger}$ whence

$$
s \cong\left[\begin{array}{ccc}
g & 0 & 0 \\
0 & 0 & (e-g) t^{*}(1-e) \\
0 & -h(e-g) t(e-g) & (1-e) t^{*}(1-e)
\end{array}\right]
$$

for some $t^{*} \in \Re$. Since

$$
R(-h(e-g) t(e-g))=R(e-g),
$$

then

$$
(-h(e-g) t(e-g))_{l}=(e-g)_{l},
$$

and by a similar argument to one above we have, for some $t^{\prime} \in \Re$,

$$
s \cong\left[\begin{array}{ccc}
g & 0 & 0 \\
0 & e-g & 0 \\
0 & 0 & (1-e) t^{\prime}(1-e)
\end{array}\right] .
$$

This useful lemma permits us to obtain an analogue in continuous rings for a diagonalization theorem of Dieudonné $[1, p .30]$.

Theorem 1. In a continuous ring $\Re$, let $e^{2}=e, R(e)<1$ and $s$ be nonsingular. Then, for some $t \in \Re$,

$$
s \cong e+(1-e) t(1-e)
$$

Proof. If $R(e)<1 / 2$, a similar proof to that of Lemma 2 yields the result.

We may suppose then, that

$$
\sum_{i=1}^{p-1} 2^{-i} \leqq R(e)<\sum_{i=1}^{p} 2^{-i}
$$

for $p>1$

Let $e_{1}=e e_{1} e$ be an idempotent of rank 1/2. Then, by Lemma 2, $t_{1} s=e_{1}$ $+\left(1-e_{1}\right) s_{1}\left(1-e_{1}\right)$ for some $t_{1} \in \Re^{\dagger}$ and $s_{1} \in \Re$. If $p>2$, we let $e_{2}=\left(e-e_{1}\right)$ $\cdot e_{2}\left(e-e_{1}\right)$ be an idempotent of rank $1 / 4$; then $e_{2}$ has normalized rank $1 / 2$ in the continuous ring $\Re\left(1-e_{1}\right)$ and $\left(1-e_{1}\right) s_{1}\left(1-e_{1}\right)$ is nonsingular in this 
ring. Hence, there exists $t_{2}$ in the group $\mathfrak{R}^{\dagger}$ of $\mathfrak{R}\left(1-e_{1}\right)$ such that

$$
t_{2}\left(1-e_{1}\right) s_{1}\left(1-e_{1}\right)=e_{2}+\left(1-e_{1}-e_{2}\right) s_{2}\left(1-e_{1}-e_{2}\right)
$$

where $s_{2} \in \Re\left(1-e_{1}\right) \subset \Re$. Then

$$
\left(e_{1}+t_{2}\right)\left(e_{1}+\left(1-e_{1}\right) s_{1}\left(1-e_{1}\right)\right)=e_{1}+e_{2}+\left(1-e_{1}-e_{2}\right) s_{2}\left(1-e_{1}-e_{2}\right) ;
$$

moreover, $e_{1}+t_{2} \in \Re^{\dagger}$ as can be verified simply.

Proceeding in a similar fashion, we have eventually, for some $s_{p-1}$ and independent idempotents $e_{i}=e e_{i} e(i=1, \cdots, p-1)$ with $R\left(e_{i}\right)=2^{-i}$

$$
s \cong e_{1}+\cdots+e_{p-1}+\left(1-e_{1}-\cdots-e_{p-1}\right) s_{p-1}\left(1-e_{1}-\cdots-e_{p-1}\right) .
$$

Application of the first statement of the proof to the idempotent $e-e_{1}$ $-\cdots-e_{p-1}$ in the subring $\Re\left(1-e_{1}-\cdots-e_{p-1}\right)$ gives

$$
\begin{aligned}
t_{p}\left(1-e_{1}-\cdots-e_{p-1}\right) s_{p-1} & \left(1-e_{1}-\cdots-e_{p-1}\right) \\
= & e-e_{1}-\cdots-e_{p-1}+(1-e) s_{p}(1-e)
\end{aligned}
$$

where

$$
t_{p} \in \mathfrak{R}\left(1-e_{1}-\cdots-e_{p-1}\right), e_{1}+\cdots+e_{n-1}+t_{p} \in \Re^{\dagger} \text { and } s_{p} \in \mathfrak{i} .
$$

The result follows.

THEOREM 2. In a continuous ring $\Omega=\Re^{\dagger}$.

Proof. The equation $u t u^{-1}=t^{2}$ is satisfied by any $t \in C^{2}$, for some $u \in\left[5\right.$ depending on $t$ [2, Theorem 2.12]. Hence the arbitrary $t \in C^{2}$ satisfies

$$
t=u t u^{-1} t^{-1}
$$

and $\Omega^{\dagger} \subseteq \Omega$.

By Lemma 2, if $a_{1}, a_{2} \in[5$ and $e$ is an idempotent such that $R(e)$ $=1 / 2$, then $a_{1}=b_{1} d_{1}, a_{2}=b_{2} d_{2}$ where $b_{1}, b_{2} \in \Omega^{\dagger}$ and

$$
d_{1}=e+(1-e) d_{1}(1-e), \quad d_{2}=e+(1-e) d_{2}(1-e) .
$$

The commutator $a_{1} a_{2} a_{1}^{-1} a_{2}^{-1}$ has the form $b d_{1} d_{2} d_{1}^{-1} d_{2}^{-1}$ with $b \in \Omega^{\dagger}$ by Corollary 2. It is sufficient to show that $d_{1} d_{2} d_{1}^{-1} d_{2}^{-1} \in \Omega^{\dagger}$ and we need only show that $d_{1} d_{2}=b^{(1)} d_{2} d_{1} b^{(2)}$ where $b^{(1)}, b^{(2)} \in \Omega^{\dagger}$. Write $(1-e) d_{1}(1-e)=\lambda$, $(1-e) d_{2}(1-e)=\mu$.

Now $e, 1-e$ define a matrix basis $s_{i j}$ with $s_{11}=e, s_{22}=1-e, s_{12}=e s_{12}$ $=s_{12}(1-e), s_{21}=(1-e) s_{21}=s_{21} e[4$, Chapter 3]. Then

$$
\left(1+s_{12}\right)\left(1-s_{21}\right)\left(1+s_{12}\right)=-s_{21}+s_{12}
$$


and

$$
\left(-s_{21}+s_{12}\right)^{2}=-s_{11}-s_{22}=-1
$$

belong to $\Omega^{\dagger}$.

Noticing that $\lambda$ has an inverse in $\Re(1-e)$ we obtain without difficulty

$$
d_{1} d_{2}=\left[\begin{array}{cc}
e & 0 \\
0 & \lambda \mu
\end{array}\right] \cong\left[\begin{array}{cc}
e & s_{12} \mu \\
0 & \lambda \mu
\end{array}\right] \cong\left[\begin{array}{cc}
e & s_{12} \mu \\
-\lambda s_{21} & 0
\end{array}\right] \cong\left[\begin{array}{cc}
0 & s_{12} \mu \\
-\lambda s_{21} & 0
\end{array}\right]
$$

and on left multiplying the last member of (4) by $-\left(-s_{21}+s_{12}\right)$

$$
d_{1} d_{2} \cong\left[\begin{array}{cc}
s_{12} \lambda s_{21} & 0 \\
0 & \mu
\end{array}\right] \cong\left[\begin{array}{cc}
0 & s_{12} \lambda \\
-\mu s_{21} & 0
\end{array}\right] \text {. }
$$

Retracting the steps of (4) we obtain the result.

REMARK 1 . When $\Re$ is a matrix ring over a field (discrete ring), $\Re, \Re^{\dagger}$ are respectively the commutator group. and the group generated by the elements of class 2. Provided the order of the matrices exceeds two, as we assume, (3) holds and again $\Omega^{\dagger} \subseteq \Re$; also $\Omega^{\dagger}$ contains the group generated by the transvections which is shown by Dieudonne [1, p. 31] to itself contain $\Re$. Hence Theorem 2 holds for rings of matrices of order greater than two.

\section{Determinants in a complete rank ring.}

DEFINITION 3. Let $\Re$ be a continuous or discrete ring. We define the determinant $\Delta(\alpha)(a \in \mathbb{F})$ as the coset $\Omega_{a}$.

We now proceed to obtain generalizations of some well-known results in determinants; the restrictions on characteristic and order apply and the determinants, we note, are defined only for nonsingular ring elements. Theorem 2, Remark 1 and the commutativity of the cosets are used freely without additional reference.

(i) A theorem on minors of the inverse.

THEOREM 3. Let $c$ be nonsingular and $e$ any idempotent in $\Re$. Then

$$
\Delta\left(1-e+e c^{-1} e\right) \Delta(c)=\Delta(e+(1-e) c(1-e)) .
$$

Proof. $\quad \Delta\left(1-e+e c^{-1} e\right) \Delta(c)=\Delta\left\{\left(1+e c^{-1}(1-e)\right)\left(1-e+e c^{-1} e\right)\right\} \Delta(c)$

$$
=\Delta((1-e) c+e)
$$




$$
\begin{aligned}
& =\Delta\{(1-(1-e) c e)((1-e) c e+(1-e) c(1-e)+e)\} \\
& =\Delta(e+(1-e) c(1-e)) .
\end{aligned}
$$

(ii) The Laplace development. (Compare [1, p. 37].)

TheoRem 4. Let $e^{2}=e, x \in \Re$. If $R(e x e)=R(e)$, then

$$
\Delta(x)=\Delta(e x e+(1-e)) \Delta(e+(1-e) x(1-e)-(1-e) x e \cdot e y e \cdot e x(1-e))
$$

where eye is the inverse of exe in $\Re(e)$.

$$
\text { Proof. } \begin{aligned}
\Delta(x)= & \Delta\{(1-(1-e) x e \cdot e y e) x\} \\
= & \Delta(e x e+e x(1-e)+(1-e) x(1-e)-(1-e) x e \cdot e y e \cdot e x(1-e)) \\
= & \Delta\{(e x e+e x(1-e)+(1-e) x(1-e)-(1-e) x e \cdot e y e \cdot e x(1-e)) \\
& \cdot(1-e y e \cdot e x(1-e))\} \\
= & \Delta(e x e+(1-e) x(1-e)-(1-e) x e \cdot e y e \cdot e x(1-e)) \\
= & \Delta(e x e+(1-e)) \cdot \Delta(e+(1-e) x(1-e) \\
& -(1-e) x e \cdot e y e \cdot e x(1-e)) .
\end{aligned}
$$

(iii) Cramer's rule.

ThEOREm 5. Let $a x=b$ be satisfied by $a, b, x \in \Re$. Then

$$
\Delta(b e+a(1-e))=\Delta(a) \Delta(e x e+(1-e))
$$

for any idempotent $e$.

Proof. $a x=b$ implies $a x e=b e$ and so

$$
\begin{aligned}
\Delta(b e+a(1-e)) & =\Delta(a x e+a(1-e)) \\
& =\Delta(a) \Delta(x e+(1-e)) \\
& =\Delta(a) \Delta\{(e x e+(1-e) x e+(1-e))(1-(1-e) x e)\} \\
& =\Delta(a) \Delta(e x e+(1-e)) .
\end{aligned}
$$

REMARK 2. The fact that Theorem 5 includes Cramer's rule can be seen as follows.

The matrix equation $A x=b$ with $A=\left(a_{i j}\right)$ an $n \times n$ matrix and $x$ $=\left\{x_{1}, \cdots, x_{n}\right\}, b=\left\{b_{1}, \cdots, b_{n}\right\}$, the components being in a field $K$, can be expressed 


$$
\left(a_{i j}\right)\left(\begin{array}{ccc}
x_{1} & & x_{1} \\
\vdots & \ldots & \vdots \\
x_{n} & & x_{n}
\end{array}\right)=\left(\begin{array}{ccc}
b_{1} & & b_{1} \\
\vdots & & \vdots \\
b_{n} & & b_{n}
\end{array}\right)
$$

where each vector is replaced by a ring element with identical columns.

Taking $e=e_{i}=\operatorname{diag}(0,0, \cdots, 1, \cdots)$ with 1 in the $i$ th place, Theorem 5 gives

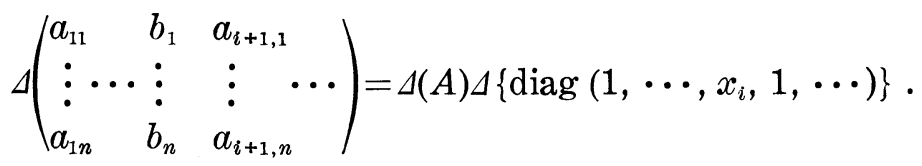

If $C$ is the commutator subgroup of $K^{\times}$, the isomorphism of $M_{n}^{\times}(K) / C_{n}$ and $M^{\times} / C$ implies the preceding equation holds when we interpret $\Delta$ as the Dieudonné determinant ( $K$ noncommutative) or as the ordinary determinant ( $K$ commutative).

\section{REFERENCES}

1. J. Dieudonné, Les déterminants sur un corps non commutatif. Bull. Soc. Math. France, 71 (1943), 27-45.

2. G. Ehrlich, Dissertation, University of Tennessee (1952).

3. J. von Neumann, papers in Proc. Nat. Acad. Sci.

(i) Continuous geometry, 22 (1936), 92-100;

(ii) On regular rings, 22 (1936), 707-713;

(iii) Algebraic theory of continuous geometries, 23 (1937), 19-22;

(iv) Continuous rings and their arithmetics, 23 (1937), 341-349.

4. Continuous geometry, Vol. II, planographed lecture notes, Institute for Advanced Study (Princeton, 1936).

Northern State Teachers College

AberdeEN, S. D. 



\title{
PACIFIC JOURNAL OF MATHEMATICS
}

\section{EDITORS}

H. L. RoYden

Stanford University

Stanford, California

\section{R. A. Beaumont}

University of Washington

Seattle 5 , Washington
A. L. Whiteman

University of Southern California

Los Angeles 7, California

E. G. Straus

University of California

Los Angeles 24, California

\section{ASSOCIATE EDITORS}

\author{
E. F. BECKENBACH \\ C. E. BURGESS \\ M. HALL \\ E. HEWITT
}
A. HORN
V. GANAPATHY IYER
R. D. JAMES
M. S. KNEBELMAN

L. NACHBIN

I. NIVEN

T. G. OSTROM

M. M. SCHIFFER
G. SZEKERES

F. WOLF

K. YOSIDA

\section{SUPPORTING INSTITUTIONS}

\author{
UNIVERSITY OF BRITISH COLUMBIA \\ CALIFORNIA INSTITUTE OF TECHNOLOGY \\ UNIVERSITY OF CALIFORNIA \\ MONTANA STATE UNIVERSITY \\ UNIVERSITY OF NEVADA \\ OREGON STATE COLLEGE \\ UNIVERSITY OF OREGON \\ UNIVERSITY OF SOUTHERN CALIFORNIA
}

Mathematical papers intended for publication in the Pacific Journal of Mathematics should be typewritten (double spaced), and the author should keep a complete copy. Manuscripts may be sent to any of the editors. All other communications to the editors should be addressed to the managing editor, E. G. Straus at the University of California, Los Angeles 24, California.

50 reprints per author of each article are furnished free of charge; additional copies may be obtained at cost in multiples of 50 .

The Pacific Journal of Mathematics is published quarterly, in March, June, September, and December. The price per volume (4 numbers) is $\$ 12.00$; single issues, $\$ 3.50$. Back numbers are available. Special price to individual faculty members of supporting institutions and to individual members of the American Mathematical Society: $\$ 4.00$ per volume; single issues, $\$ 1.25$.

Subscriptions, orders for back numbers, and changes of address should be sent to Pacific Journal of Mathematics, 2120 Oxford Street, Berkeley 4, California.

Printed at Kokusai Bunken Insatsusha (International Academic Printing Co., Ltd.), No. 10, 1-chome, Fujimi-cho, Chiyoda-ku, Tokyo, Japan.

PUBLISHED BY PACIFIC JOURNAL OF MATHEMATICS, A NON-PROFIT CORPORATION

The Supporting Institutions listed above contribute to the cost of publication of this Journal, but they are not owners or publishers and have no responsibility for its content or policies. 


\section{Pacific Journal of Mathematics}

\section{Vol. 7, No. 4 \\ April, 1957}

Robert Geroge Buschman, A substitution theorem for the Laplace

transformation and its generalization to transformations with

symmetric kernel .

S. D. Conte, Numerical solution of vibration problems in two space variables ...................................... 1535

Paul Dedecker, A property of differential forms in the calculus of variations ......................................... 1545

H. Delange and Heini Halberstam, A note on additive functions . . . . . . . 1551

Jerald L. Ericksen, Characteristic direction for equations of motion of non-Newtonian fluids ................................. 1557

Avner Friedman, On two theorems of Phragmén-Lindelöf for linear elliptic and parabolic differential equations of the second order ........... 1563

Ronald Kay Getoor, Additive functionals of a Markov process . . . . . . . . . 1577

U. C. Guha, $(\gamma, k)$-summability of series . . . . . . . . . . . . . . . . . . 1593

Alvin Hausner, The tauberian theorem for group algebras of vector-valued functions ...................................... 1603

Lester J. Heider, $T$-sets and abstract $(\mathrm{L})$-spaces ................. 1611

Melvin Henriksen, Some remarks on a paper of Aronszajn and

Panitchpakdi........................................ 1619

H. M. Lieberstein, On the generalized radiation problem of A. Weinstein ... 1623

Robert Osserman, On the inequality $\Delta u \geq f(u) \ldots \ldots \ldots \ldots \ldots \ldots \ldots . \ldots 1641$

Calvin R. Putnam, On semi-normal operators . . . . . . . . . . . . . . . . . . 1649

Binyamin Schwarz, Bounds for the principal frequency of the

non-homogeneous membrane and for the generalized Dirichlet integral...

Edward Silverman, Morrey's representation theorem for surfaces in metric

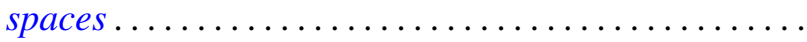

V. N. Singh, Certain generalized hypergeometric identities of the Rogers-Ramanujan type. II .

R. J. Smith, A determinant in continuous rings 1701

Drury William Wall, Sub-quasigroups of finite quasigroup 1711

Sadayuki Yamamuro, Monotone completeness of normed semi-ordered linear spaces.

C. T. Rajagopal, Simplified proofs of "Some Tauberian theorems" of Jakimovski: Addendum and corrigendum ............

N. Aronszajn and Prom Panitchpakdi, Correction to: "Extension of uniformly continuous transformations in hyperconvex metric spaces"...................................

Alfred Huber, Correction to: "The reflection principle for polyharmonic functions" 